\title{
Repair of Soft Tissue Defect with Intraoperative Tissue Expansion after Surgical Resection of Dermatofibrosarcoma Protuberans: A Case Report
}

\author{
Jin Soo Kim¹ (D), llou Park (D), Sung Hoon Koh¹ (D), Dong Chul Lee ${ }^{1}$ (D), Hee Jeong Lee² (D) \\ Departments of ${ }^{1}$ Plastic and Reconstructive Surgery and ${ }^{2}$ Pathology, Gwangmyeong Sungae General Hospital, Gwangmyeong, Korea
}

\begin{abstract}
While it is the most frequently observed subtype of all cutaneous soft tissue sarcomas, dermatofibrosarcoma protuberans is still uncommon, with a high local recurrence rate. Although surgical resection could be a simple curative procedure, surgeons can encounter difficulties when dissecting or closing the wound, depending on the location and the size of the tumor. We present a case report of direct repair of soft tissue defect with intraoperative tissue expansion after sarcoma resection. A 62-year-old male visited our clinic with an approximately $1.5 \times 1.5 \mathrm{~cm}$-sized mass on his lower leg. We excised the mass, which a pathologic study confirmed as dermatofibrosarcoma protuberans. The authors planned a wide excision of the lesion as soon as possible. The excised lesion was not large; however, the soft tissue defect could not be closed by direct approximation. We eventually closed the tumor resection wound using a Foley catheter as a tissue expander. The patient's wound healed well, albeit leaving a minimal scar. This case suggests that a Foley catheter could be an effective and versatile tool that is readily available in any medical setting, including after a small tumor resection in outpatient surgery.
\end{abstract}

Keywords: Dermatofibrosarcoma protuberans; Tissue expansion; Foley catheter

\section{Introduction}

Dermatofibrosarcoma protuberans (DFSP) is an uncommon malignant cutaneous fibroblastic neoplasm [1]. Although the local recurrence rate of DFSP is high, it displays slow infiltrative growth and little metastatic potential. Its clinical features are initial plaque-like dermal thickening and progression to a raised firm multinodular mass [2]. Typical microscopic findings are characterized by infiltrative storiform fascicles of uniform bland spindle cells, with diffuse CD34 expression [3,4]. Some patients with DFSP may be associated with adenosine deaminase-deficient severe combined immunodeficiency [5]. The optimal therapy is complete surgical resection, similar to treatment of other cutaneous malignancies.

However, surgeons sometimes struggle to close the surgical wound after resection if its size is large or its location does not afford sufficient soft tissue; such locations include the head, joints, and some areas adjacent to bones. Herein, we report a successful case of primary closure with intraoperative tissue expansion after wide excision of DFSP. This study was approved by the Institutional Review Board of Gwangmyeong Sungae General Hospital (No. KIRB-2021-N-003) and performed in accordance with the principle of the Declaration of Helsinki. The patient provided written informed consent for the publication and the use of his images.
Case Report

Received: June 25, 2021

Revised: September 24, 2021

Accepted: September 25, 2021

Corresponding author:

Sung Hoon Koh, M.D., Ph.D.

Department of Plastic and Reconstructive Surgery, Gwangmyeong Sungae General Hospital,

36 Digital-ro, Gwangmyeong 14241, Korea

Tel: +82-2-2680-7637

Fax: +82-2-2615-7218

E-mail: pshkoh@hanmail.net

This is an Open Access article distributed under the terms of the Creative Commons Attribution Non-Commercial License (https://creativecommons org/licenses/by-nc/4 O/) which permits unrestricted non-commercial use, distribution, and reproduction in any medium, provided the original work is properly cited.

C) 2021 Korean Wound Management Society 


\section{Case}

A 62-year-old man visited our clinic with a protruding mass $1.5 \times 1.5 \mathrm{~cm}$ in size on his right lower leg. It had insidious onset about 6 months prior, and the patient was healthy without any underlying disease. He did not have any special family history, either. All vital signs were stable and there was no palpable lymphadenopathy. Clinical inspection showed a firm round mass with mild pigmentation approximately at the distal third level of the fibula (Fig. 1). The mass was totally excised under local anesthesia in an outpatient operating room, and the gross specimen was observed to be a benign tumor, such as granuloma or dermatofibroma (Fig. 2). However, the histopathologic study revealed spindle cells with a storiform and infiltration to fibrous septa, which was diagnosed as DFSP (Fig. 3).

After confirming nonspecific findings in chest, abdomen, and extremities computed tomography scans, we made a plan

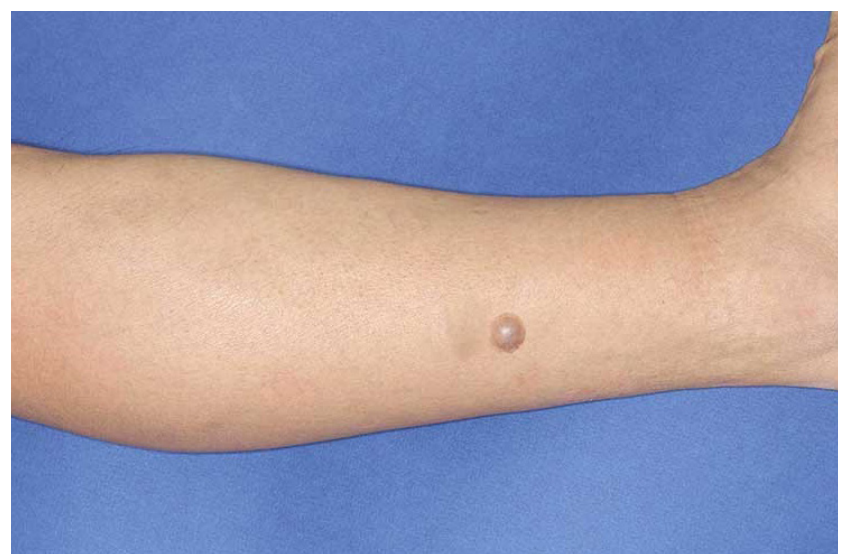

Fig. 1. Preoperative photograph on the first visit. A brownish papule is observed approximately at the distal third of the fibula.

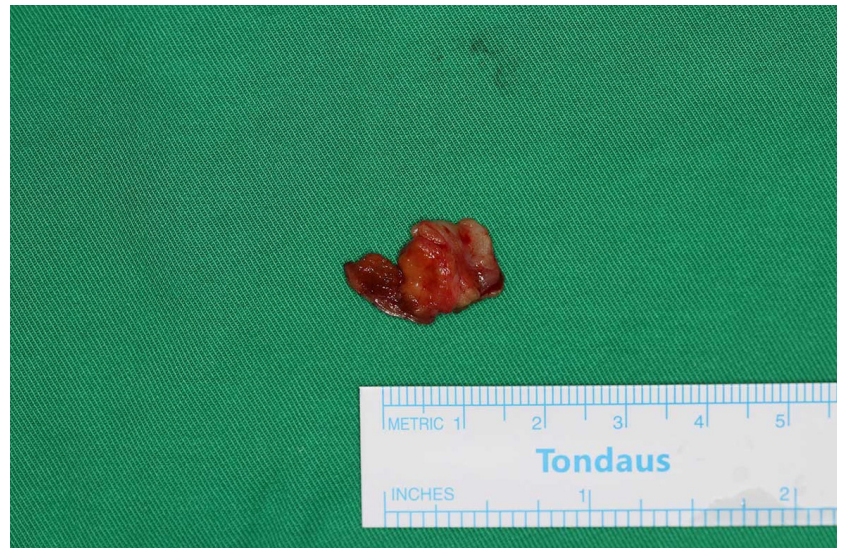

Fig. 2. Gross specimen after the first excision. The mass was histologically diagnosed as dermatofibrosarcoma.
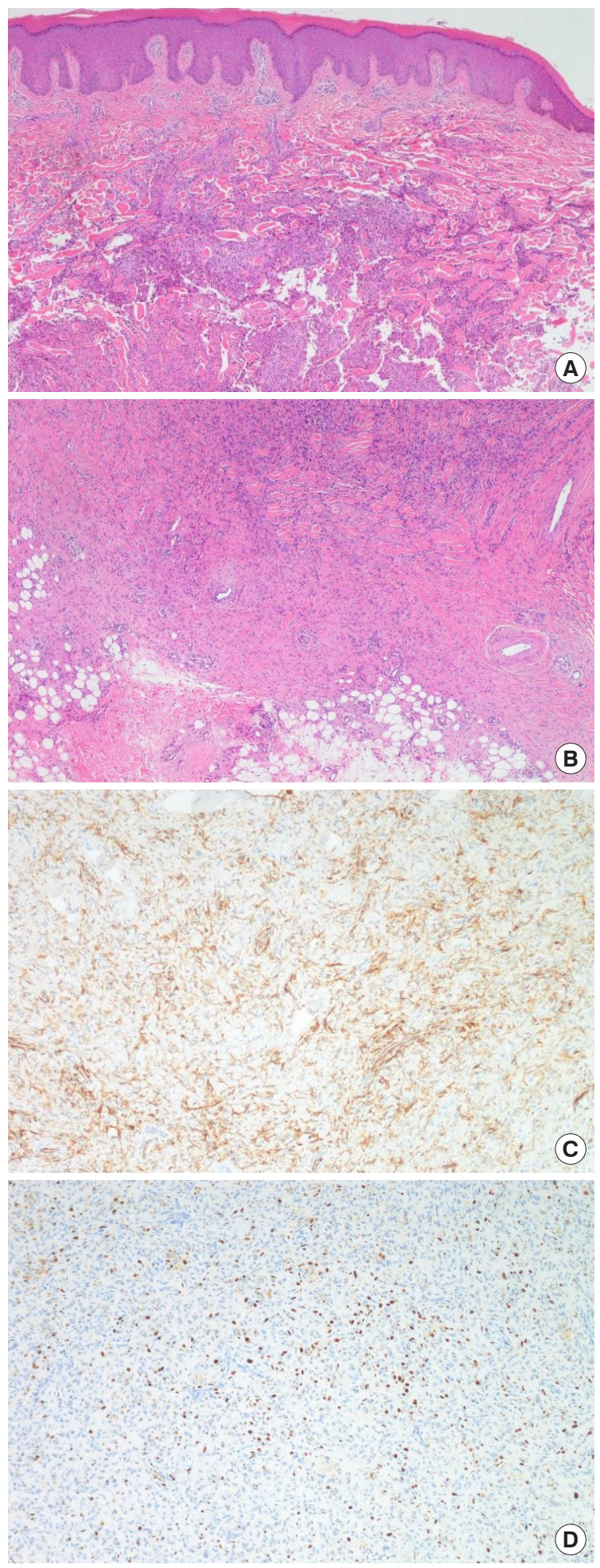

Fig. 3. Microscopic images of the mass. (A) Spindle cells with a storiform to whorled pattern within dermis (H\&E, $\times 40)$. (B) Infiltration into fibrous septa with a honeycomb pattern (H\&E, $\times 40)$. (C) Diffuse expression of CD34 (CD34 staining, $\times 100$ ). (D) Increased Ki-67 expression-a marker of cellular proliferation (Ki-67 staining, $\times 100)$. 
to widely excise the previous surgical wound 2 weeks later. We expected an approximately 2 to $3 \mathrm{~cm}$-sized further excision to be required around the previous excision wound. Under local anesthesia, the earlier sutured wound was excised with negative margin in frozen biopsy. The size of the excised lesion was $3.5 \times 2 \times 0.8 \mathrm{~cm}$ (Fig. $4 \mathrm{~A}$ ). It was relatively small; however, the lesion was adjacent to the distal third level of the peroneus longus muscle and tibia, which did not afford sufficient soft tissue to close after resection. Thus, we put a Foley catheter into the
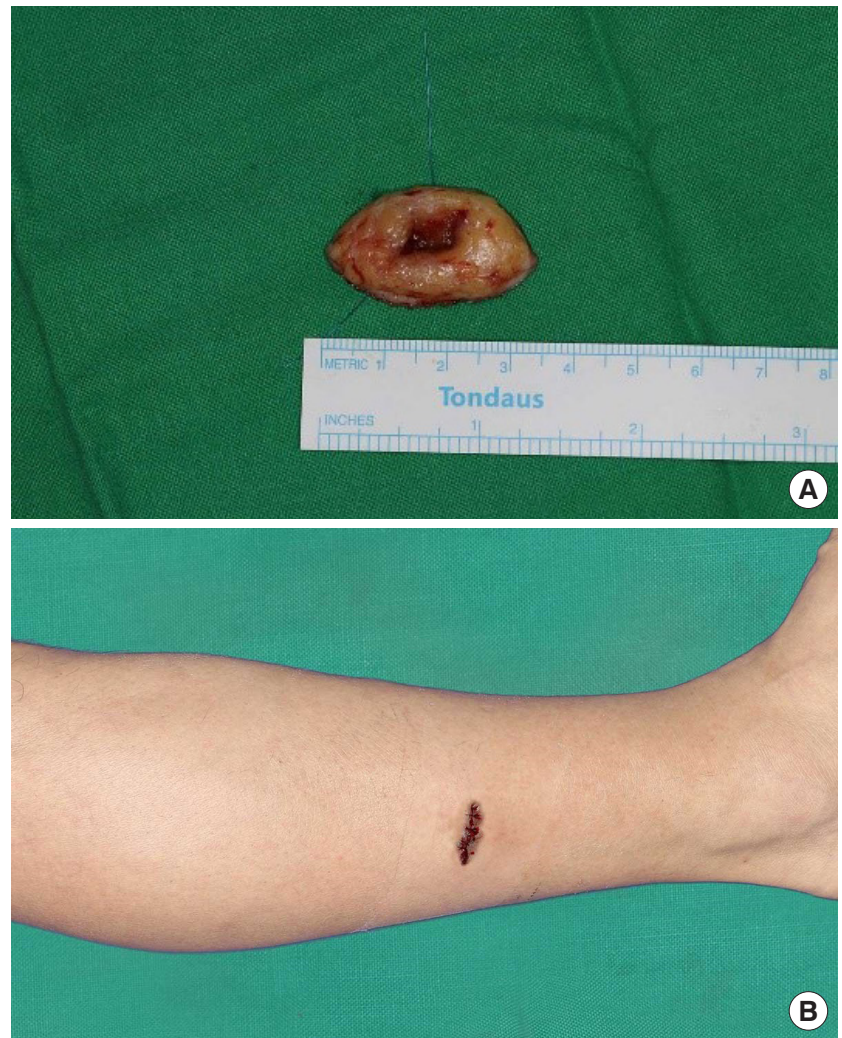

Fig. 4. Gross specimen and immediate postoperative photographs after wide excision. (A) The size of excision is approximately $3.5 \times 2 \times 0.8 \mathrm{~cm}$. (B) The surgical wound is closed completely without any local circulatory problems.

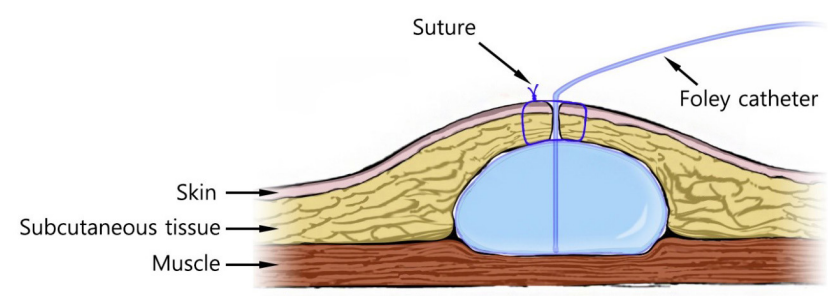

Fig. 5. Illustration of the tissue expansion. The surgical pocket is temporarily sutured after inserting a Foley catheter. pocket of the excised wound for intraoperative tissue expansion after sufficient undermining. The incision wound was closed with 3-0 nylon sutures to prevent the Foley catheter from extruding, and the Foley catheter was inflated with $5 \mathrm{~mL}$ of normal saline twice ( 5 minutes followed by 10 minutes, with 5 minutes of intermittent rest period) until the local skin blanching had disappeared (Fig. 5). If the empty space left by resection is in a relatively deep subcutaneous layer, the Foley catheter will be inflated evenly, and the sutured skin will not have too much tension. We checked the capillary refilling around the skin again and observed only marginal bleeding through the remnant subcutaneous tissue after removal of the Foley catheter. The subcutaneous layer was then sutured with 4-0 Vicryl, and the skin was closed with 4-0 nylon (Fig. 4B). The wound was dressed with floppy gauzes and an elastic bandage.

The skin sutures were removed on postoperative day 14 , and the pathologic report confirmed all negative margins around the circumferential and deep resection. Any complications such as hematoma, dehiscence, and necrosis were not found; there was no minor scarring, and no recurrence on follow-up after 10 months (Fig. 6).

\section{Discussion}

DFSP is a rare cutaneous malignancy clinically characterized by an exophytic nodular mass with skin discoloration, ranging from 1 to $30 \mathrm{~cm}$. It is the most common dermal sarcoma, and typically arises between 20 to 59 years of age [1,2]. DFSP is a low-grade sarcoma with slow infiltration and little metastatic potential; however, it has a high rate of local recurrence after surgical excision. Most occur on the trunk (40\%-50\%), fol-

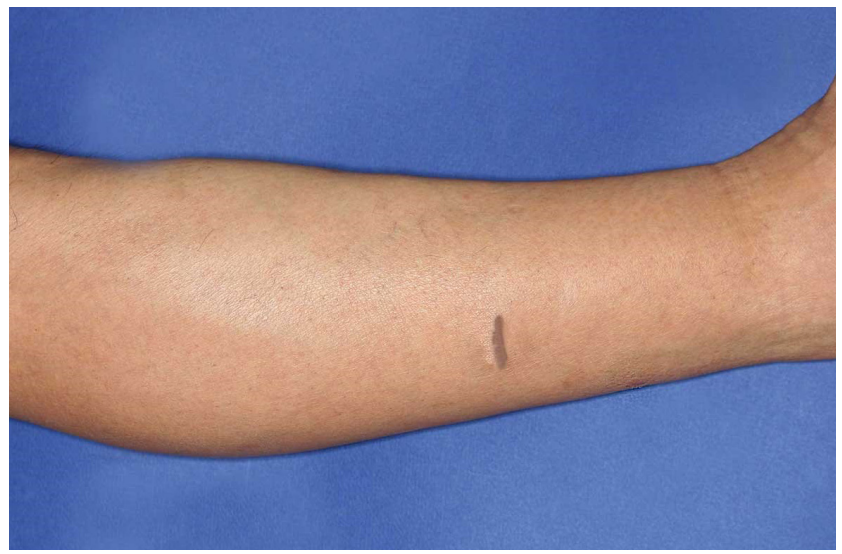

Fig. 6. Long-term follow-up photograph (10 months). No complications had occurred, and only a minor scar is observed. 
lowed by the proximal extremities (30\%-40\%), and head and neck (10\%-15\%) [2-4]. In molecular studies, DFSP is characterized by a specific $\mathrm{t}(17 ; 22)(\mathrm{q} 22 ; \mathrm{q} 13)$ translocation leading to a fusion of collagen type I alpha 1 and platelet-derived growth factor subunit $B[5,6]$. Typical microscopic findings are spindle cells with a storiform to whorled pattern, minimal cytological atypia, a low mitotic count within a collagenous stroma, infiltration to fibrous septa (known as the honeycomb pattern), and strong and diffuse expression of CD34 [3,7]. Some patients with DFSP may be associated with adenosine deaminase-deficient severe combined immunodeficiency. As its clinical features are not sufficiently specific for a diagnosis, care should be taken to differentiate it from dermatofibroma, fibrosarcoma, pleomorphic sarcoma of the skin, leiomyosarcoma, malignant peripheral nerve sheath tumors, rare variants of spindle cell malignant melanoma, atypical fibroxanthoma, and nodular fasciitis $[8,9]$. Although systemic therapy with imatinib is needed in the progressive stage, the first-line treatment is complete surgical resection, the same as treatment of other cutaneous malignant neoplasms [10].

DFSP is difficult to distinguish from dermatofibroma based on visual appearance. If fibrosarcomatous transformation occurs, it has high risk of recurrence and metastasis [10]. Although DFSP is a rare sarcoma, it is still the most frequent among cutaneous sarcomas. Thus, even a small mass-like dermatofibroma should be thoroughly inspected, and surgeons should be suspicious of malignancy in early to middle adulthood.

Procedures and surgeries using tissue expansion have been developed in the reconstruction of diverse skin and soft tissue defects since it was first introduced in 1957 [11]. The expanded tissue induces adjacent skin migration, cell proliferation, and angiogenesis. Histophysiologic studies have revealed a numerical increment of dermal fibroblasts, increased epidermal mitotic activity and thickened elastic fibers in expanded skin [12]. In general, a tissue expansion procedure consists of three steps: expander insertion, expander inflation for several weeks, and expander removal. The intraoperative tissue expansion (ITE) is a modified technique to rapidly and immediately expand certain areas with previously limited expansion [13]. Unlike conventional tissue expansion, mechanical deformation of the ITE is noted by four factors: (1) displacement of fluids and ground substance out of the collagen network leads to relative dehydration of tissue; (2) collagen fibers realign in a parallel fashion; (3) elastic fibers microfragment; and (4) adjacent tissue migrates into the expanded field as a result [12].
Even though tumor resection itself could be a simple procedure, surgeons occasionally confront situations where they are to close excisional wounds with insufficient soft tissue. In such circumstances, there are several options to close the wound, such as a local flap, a pedicled flap, or a free flap, etc. For a large defect, this type of surgery can be indispensable. However, for small defects or outpatient surgeries, such a procedure would place an unnecessary burden not only on patients but also surgeons. Therefore, we tried to close the wound with a more simple procedure-the ITE.

The incision for tissue expansion should usually be performed on the lateral side of the lesion or a distant location from the area to be expanded. Afterwards, the site should be undermined, and when the tissue expander is inserted through the incision wound, it will be expanded diffusely. However, in this case, after wide tumor resection, the temporarily repaired incision was positioned in the center of the lesion. Although the expansion was not done evenly down to the deep subcutaneous level, a large expansion more than several centimeters was not necessary. Hence, the tissue was expanded as much as needed to close the surgical incision with en bloc sutures in skin and superficial fat tissue layer.

Among various ITE instruments, a Foley catheter could be a readily available, economical, useful and safe device employed in an easy procedure without any circulatory complications $[13,14]$. In addition, it is so easy to procure in any medical setting that it can enable surgeons to repair the surgical wound primarily even in an outpatient operating room. While the Foley catheter cannot be applied to large tissue defects, this case of reconstruction after malignant tumor resection could be an example where "a stitch in time saves nine."

\section{Conflict of interest}

No potential conflict of interest relevant to this article was reported.

\section{ORCID iDs}

Jin Soo Kim

Ilou Park

Sung Hoon Koh

Dong Chul Lee

Hee Jeong Lee https://orcid.org/0000-0003-3369-2974 https://orcid.org/0000-0002-6856-4895 https://orcid.org/0000-0002-8094-2561 https://orcid.org/0000-0003-4211-6901 https://orcid.org/0000-0003-0053-5665 


\section{References}

1. Criscione VD, Weinstock MA. Descriptive epidemiology of dermatofibrosarcoma protuberans in the United States, 1973 to 2002. J Am Acad Dermatol 2007;56:968-73.

2. Chang CK, Jacobs IA, Salti GI. Outcomes of surgery for dermatofibrosarcoma protuberans. Eur J Surg Oncol 2004;30:341-5.

3. Noujaim J, Thway K, Fisher C, et al. Dermatofibrosarcoma protuberans: from translocation to targeted therapy. Cancer Biol Med 2015;12:375-84.

4. Reha J, Katz SC. Dermatofibrosarcoma protuberans. Surg Clin North Am 2016;96:1031-46.

5. Kesserwan C, Sokolic R, Cowen EW, et al. Multicentric dermatofibrosarcoma protuberans in patients with adenosine deaminase-deficient severe combined immune deficiency. J Allergy Clin Immunol 2012;129:762-9.

6. Bowne WB, Antonescu CR, Leung DH, et al. Dermatofibrosarcoma protuberans: a clinicopathologic analysis of patients treated and followed at a single institution. Cancer 2000;88:2711-20.

7. García JJ, Folpe AL. The impact of advances in molecular genetic pathology on the classification, diagnosis and treatment of selected soft tissue tumors of the head and neck.
Head Neck Pathol 2010;4:70-6.

8. Sirvent N, Maire G, Pedeutour F. Genetics of dermatofibrosarcoma protuberans family of tumors: from ring chromosomes to tyrosine kinase inhibitor treatment. Genes Chromosomes Cancer 2003;37:1-19.

9. Bogucki B, Neuhaus I, Hurst EA. Dermatofibrosarcoma protuberans: a review of the literature. Dermatol Surg 2012;38:537-51.

10. Saiag P, Grob JJ, Lebbe C, et al. Diagnosis and treatment of dermatofibrosarcoma protuberans: European consensusbased interdisciplinary guideline. Eur J Cancer 2015;51: 2604-8.

11. Margulis A, Billig A, Elia J, et al. Complications of postburn tissue expansion reconstruction: 9 years experience with 42 pediatric and 26 adult patients. Isr Med Assoc J 2017;19:100-4.

12. Johnson TM, Lowe L, Brown MD, et al. Histology and physiology of tissue expansion. J Dermatol Surg Oncol 1993;19:1074-8.

13. Greenbaum SS. Intraoperative tissue expansion with the Foley catheter. J Dermatol Surg Oncol 1993;19:1079-83.

14. Funakoshi Y, Shono T, Kurogi A, et al. Intraoperative tissue expansion using a foley catheter for a scalp defect: technical note. World Neurosurg 2020;143:62-7. 